\title{
AUTOMATIC DETECTION OF ROAD EDGES FROM AERIAL LASER SCANNING DATA
}

\author{
L. Truong-Hong ${ }^{1}$, D.F. Laefer ${ }^{2}$, R.C. Lindenbergh ${ }^{1}$ \\ ${ }^{1}$ Dept. of Geoscience \& Remote Sensing, Delft University of Technology, Delft, The Netherland, - (1.truong; \\ r.c.lindenbergh)@tudelft.nl \\ ${ }^{2}$ Center for Urban Science and Progress, New York University, New York, USA - debra.laefer@nyu.edu
}

Commission II, WG II/3

KEY WORDS: Road Curb, Road Extraction, Aerial Laser Scanning, Cell Decomposition, Cell-based Region Growing

\begin{abstract}
:
When aerial laser scanning (ALS) is deployed with targeted flight path planning, urban scenes can be captured in points clouds with both high vertical and horizontal densities to support a new generation of urban analysis and applications. As an example, this paper proposes a hierarchical method to automatically extract data points describing road edges, which are then used for reconstructing road edges and identifying accessible passage areas. The proposed approach is a cell-based method consisting of 3 main steps: (1) filtering rough ground points, (2) extracting cells containing data points of the road curb, and (3) eliminating incorrect road curb segments. The method was tested on a pair of $100 \mathrm{~m}$ x 100m tiles of ALS data of Dublin Ireland's city center with a horizontal point density of about 325 points $/ \mathrm{m}^{2}$. Results showed the data points of the road edges to be extracted properly for locations appearing as the road edges with the average distance errors of $0.07 \mathrm{~m}$ and the ratio between the extracted road edges and the ground truth by $73.2 \%$.
\end{abstract}

\section{INTRODUCTION}

Digital road networks are useful for many urban applications: urban planning, disaster management, virtual tourism, and autonomous navigation, among others. An accurate digital road can also assist users to maximize safe driving conditions and road authorities in effective asset management. As part of achieving that, the road edge is an important feature for identifying the road area. While road surfaces and/or centrelines have been extracted from various forms of imagery (Lacoste et al., 2005, Lafarge et al. 2010; Seibert et al. 2013), accurate and complete roads can be only resolved with high quality images with sufficient and accurate geometric information of objects. That is because the images are captured by passive sensors strongly affected by weather conditions (e.g. sunlight or frog), and a scene with obstruction often causes occlusions and shadows.

Laser scanning technology offers an alternative to capturing a road surface and its scene from various positions (e.g. ground and air). Although terrestrial and mobile laser scanning (TLS and MLS) data are highly dense, accurate and widely using for road extraction (Pu et al., 2011), aerial laser scanning (ALS) data have been limited for such applications because of sparse and low accuracy. However, with a special flight planning, a horizontal point density exceeding 335 points $/ \mathrm{m}^{2}$ for a multi-pass flight mission can be easily achieved (Laefer et al., 2017). With such a point density, ALS data can sufficiently describe the geometry of a road environment to recognize and reconstruct road objects including the road shoulder, sides, and markings, as well as light poles, traffic signs, and crash barriers.

In processing point clouds for road extraction and reconstruction, road edges can be extracted from entire point clouds by using changes in elevation, point orientation, and intensity values of data points, or from data points on boundaries of the road surface (Clode et al., 2004; Vo et al., 2015). However, robust automatic extraction and reconstruction of road edges from ALS data is still difficult because of incomplete data sets and the diversity and complexity of the road networks and surrounding scenes. When target flight path planning (Hinks et al., 2009) is combined with a new hierarchical algorithm, an appropriate data set and workflow can be achieved to enable automatic road edge reconstruction in urban areas from dense ALS data.

\section{RELATED WORKS}

Satellite and aerial images and laser scanning data have been widely used to extract and reconstruct road edges and surfaces. Image-based approaches have been developed by many researchers (e.g. Ferchichi et al., 2005; Seibert et al., 2013; Zhu et al., 2004). This section is restricted to LiDAR-based methods, as a systematic overview of techniques using images for road detection was recently published elsewhere (i.e. Wang et al., 2016).

Most of methods for extracting the road edges start by separating the road edge points from the entire LiDAR dataset by using various thresholds such as those based on elevation, point orientation or intensity values of the data points. For example, Vosselman et al. (2009) proposed three steps to detect kerbstones from ALS data including: (i) extracting a point cloud near terrain surface describing the kerbstones by height jump criteria computing from neighbour points, (ii) generating the middle points between high and low points in a set of the height jump points, and (iii) fitting these middle points with a smooth curve and closing small gaps between nearby and collinear line segments. The work proved that ALS data with a resolution as little as 20 points $/ \mathrm{m}^{2}$ was a feasible resource to extract the road side but that parked cars were a major obstacle to obtaining data points along the curb. To improve the accuracy of the middle points for that technique, Zhou et al. (2012) introduced a least square fitting of a sigmoidal function describing the elevation (zcoordinates) of the height jump points.

By deploying MLS data for road extraction, Jaakkola et al. ( 2008) introduced an automatic method for finding a point cloud of the curb stones from the height image along the scanned profile. Similarly, Guan et al. (2014) extracted road curbs based 
on the calculated slope between two consecutive points and the elevation difference of a given point to its neighbourhood in the scan line. Moreover, Kumar et al. (2013) added pulse width attributes to the elevation and reflectance data with an MLS data set to distinguish the road surface from the grass-soil and the kerb edges for rural roads. The approach combined a gradient vector flow and balloon parametric active contour models. Also using elevation differences, point density and slope change, Yang et al. (2013) detected curb points after employing a moving window operator to filter non-ground points from a point cloud of road cross-sections. The study demonstrated a high sensitivity to the selected length of the moving window. Moreover, based on a range image from the point cloud, Serna et al. (2013) used height in a range from $3 \mathrm{~cm}$ to $20 \mathrm{~cm}$ and geodetic elongation by 10 to detect curb points. However, occlusions and long access ramps can be the main problems in detecting the curbs.

In another direction, the point cloud of road surface has been extracted using morphology or segmentation-based methods. In such approaches, typically the curb stones are extracted from primitives of the road surfaces. For example, Ibrahim et al. (2012) segmented a road surface from MLS data based point density respecting the distance from the vehicle's trajectory and using a morphological analysis. Then, a 3D edge detection algorithm based on a derivative of a Gaussian function was employed to extract the curb points. Another segmentation-based method to extract a point cloud of road surfaces was introduced by Smadja et al. (2010), who used a RANSAC-based algorithm to extract rough road segmentation. That technique demonstrated that curb boundaries could be extracted, if the slope break of the points was larger than the slope threshold of $30^{\circ}$. The curb points were subsequently used to estimate the road width and a road edge by using a spline arc-length NURBS estimation. Similarly, Qui et al. (2016) also used a RANSAC-based algorithm to segment a point cloud of a road surface by having the normal vector less than threshold angle. Next, the best candidate road edge points were extracted by comparing the energy function about the smoothness. Subsequently, these edge points were refined by using road width and continuity. Additionally, Miraliakbari et al. (2015) proposed two region growing strategies based on height difference and histogram-jump detection to extract a road surface, and splines were used to approximate final road boundaries.

In summary, although several methods have been developed to detect point clouds of road edge, such methods seemly fail to provide an efficient and robust solution because they require several a priori knowledge of the local environment and/or as empirical input parameters. Additionally, they are computationally expensive as the methods compute multiple features (e.g. a slope, gradient or height difference) for each data point. In response to these drawbacks, the paper introduces a computationally efficient and robust method for road extraction from ALS data.

\section{PROPOSED METHOD}

The underlying idea behind the proposed method is that road curb points are located on boundaries between a road surface and a footpath and are distributed along the road direction. The proposed method shown in Fig. 1 consists of three main steps: (1) Step 1: filtering ground points, (2) Step 2: extracting and grouping cells containing data points of the road edges, and (3) Step 3: eliminating incorrect road edge segments. The proposed method first employs a quadtree to recursively subdivide an initial bounding box of ALS data points into smaller cells until a terminal condition of a minimum cell size is reached. A cell is then classified as "full", if the cell contains at least one sample point; otherwise it classified as "empty". Each full cell has an attribute called the cell height, the maximum difference of the $\mathrm{z}$ coordinates of the data points within the cell.
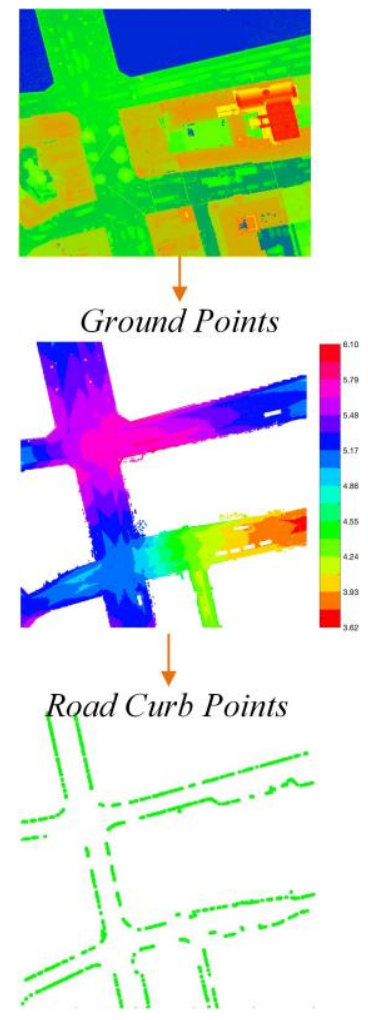

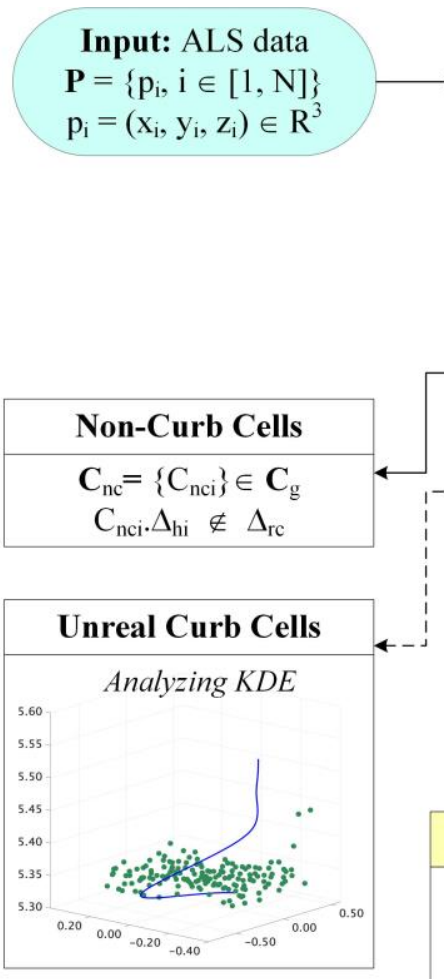

Figure 1. Workflow of the proposed method
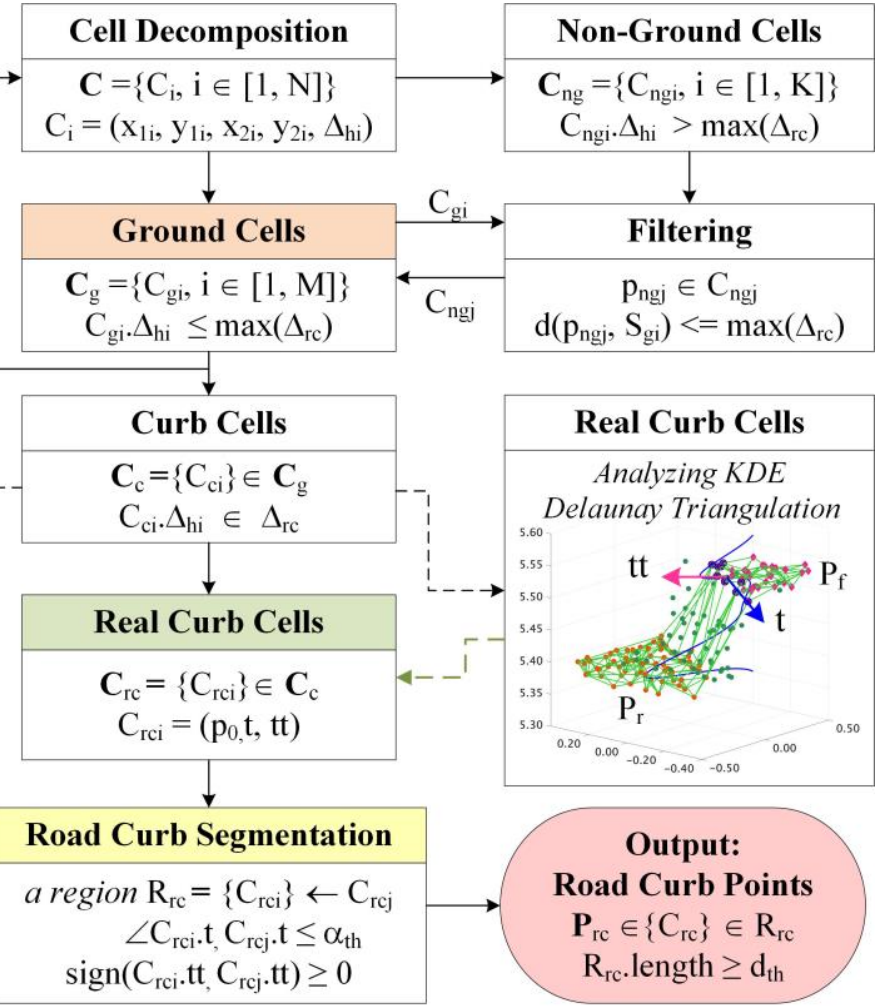

$\mathrm{R}_{\mathrm{rc}}$. length $\geq \mathrm{d}_{\mathrm{th}}$ 
As point clouds of road edges are located on the boundary between a footpath and a road surface, the road edge points are commonly within cells containing points of both the footpath and the road. This observation implies that if a cell contains the road edge points, the cell height should be that of the curb height. As such, in Step 1, full cells are classified as ground and non-ground cells $\left(\mathbf{C}_{\mathrm{g}}\right.$ and $\left.\mathbf{C}_{\mathrm{ng}}\right)$ based on the cell height. In practice, the curb height $\left(\Delta_{\mathrm{rc}}\right)$ varies commonly in a range from $10 \mathrm{~cm}$ to $30 \mathrm{~cm}$. The maximum curb height $\left(\Delta_{\mathrm{rc}, \max }\right)$ of $30 \mathrm{~cm}$ was adopted as the threshold for this classification. For example, with an input data points in Fig. 2a, a cell was generated and classified as ground or non-ground as shown in Fig. $2 b$.

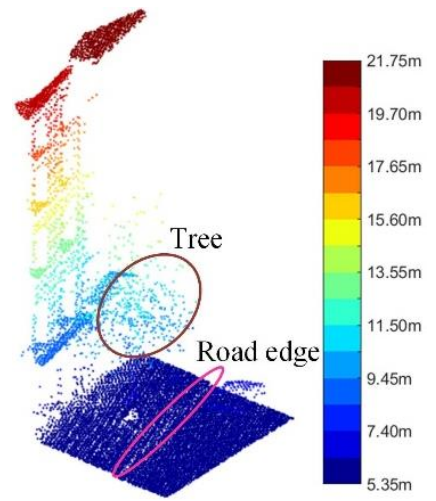

a) Point cloud containing data points of a tree, building, road, curb, and footpath

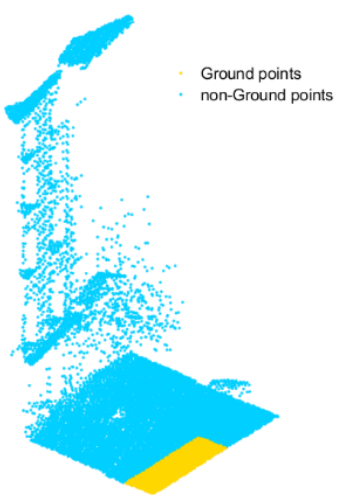

c) Ground and non-ground points before refinement

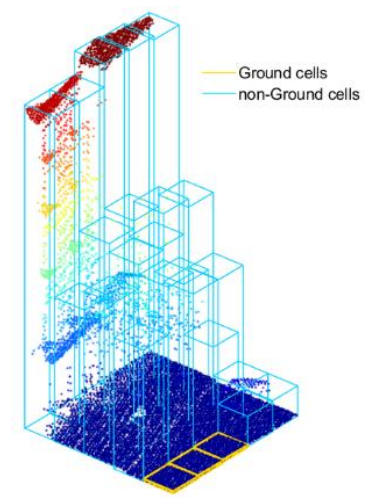

b) Cell classification based on cell height

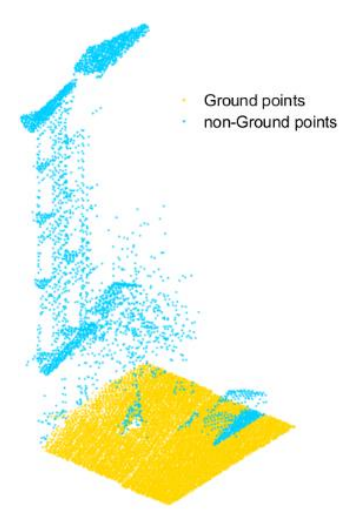

d) Ground and non-ground points after refinement
Figure 2. Extract cell-based ground point extraction

However, at locations of trees or light poles (e.g. Fig. 2a), the cell containing the road edge points can classified as $\mathbf{C}_{\mathrm{ng}}$ (Fig. 2b). In this case, some road edge points may be excluded from the ground points, because of the presence of street furniture (Fig. 2c). Thus, a surface-based filtering technique was implemented to re-extract the ground points from the non-ground cells, $\mathbf{C}_{\mathrm{ng}}$. For this task, from each $\mathbf{C}_{\text {ngi }} \in \mathbf{C}_{\text {ng }}$ adjoined to $\mathrm{C}_{\mathrm{gi}} \in \mathbf{C}_{\mathrm{g}}$, points $\mathrm{q}_{\mathrm{i}} \in \mathbf{C}_{\text {ngi }}$ are considered as the ground points, if the distance $\mathrm{d}\left(\mathrm{q}_{\mathrm{i}}\right.$ $\in \mathrm{C}_{\mathrm{ngi}}, \mathrm{S}_{\mathrm{gi}}$ ) was less than $\Delta_{\mathrm{rc} \text {,max }}$, in which $\mathrm{S}_{\mathrm{gi}}$ is a fitting plane of all points of $\mathrm{C}_{\mathrm{gi}}$ by using a principal component analysis (PCA). The $q_{i}$ were kept in $C_{n g i}$ while other points were removed from the $\mathrm{C}_{\text {ngi }}$ cells (Fig. $2 \mathrm{~d}$ ). Each $\mathrm{C}_{\text {ngi }}$ cell was then re-labelled as the ground cell, if the cell possessed at least 5 ground points.

As mentioned above, the road edge points are often located in cells containing points from both the footpath and the road surface, which implies the height of the cell should be in the range of the curb height. As such, in Step 2 the $\mathbf{C}_{\mathrm{g}}$ cells were first divided into non-curb cells $\left(\mathbf{C}_{\mathrm{nc}}\right)$ and curb cells $\left(\mathbf{C}_{\mathrm{c}}\right)$ based on the curb height, with $\Delta_{\mathrm{rc}}$ varying from $10 \mathrm{~cm}$ to $30 \mathrm{~cm}$. However, due to errors in data acquisition, registration and noise data, although $\mathbf{C}_{\mathrm{c}}$ satisfies the cell height condition, the cell may not contain data points of the road edges. By analysing a distribution of the point cloud within $\mathbf{C}_{c}$ in an elevation direction (or $\mathbf{z}$ axis), cell possessing both footpath and road surface points exhibit two peaks when the kernel density curve is derived (Fig. 3). Thus, the kernel density estimation (KDE) (Laefer et al., 2017) was employed to analyse a distribution of $\mathrm{z}$-coordinates of the point cloud within $\mathbf{C}_{\mathrm{c}}$ to divide $\mathbf{C}_{\mathrm{c}}$ cells into the real curb cells $\left(\mathbf{C}_{\mathrm{rc}}\right)$ and unreal curb cells $\left(\mathbf{C}_{\mathrm{uc}}\right.$ ). The bandwidth (bw) of 5 times the average sampling step of the points within $\mathbf{C}_{\mathrm{c}}$ in the elevation was adopted to generate the kernel density curve.

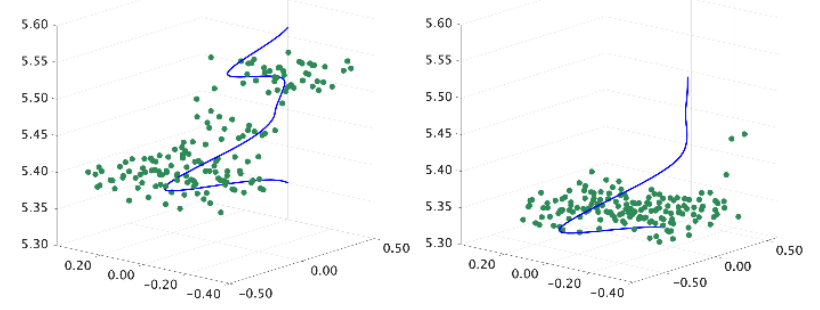

a) Available road edge points within $\mathbf{C}_{c}$ b) No road edge points within $\mathrm{C}_{\mathrm{c}}$
Figure 3. KDE of z-coordinates of the points within $\mathbf{C}_{\mathrm{c}}$

Next, the points describing the footpath and road surface were extracted based on the KDE. For this task, for each $\mathbf{C}_{\mathrm{rc}}$, two peaks on the outmost KDE were first extracted. Then, points within the buffer, [-bw/2, bw/2] of the peak position are extracted and known that the points of the footpath $\left(\mathbf{P}_{\mathrm{f}}\right)$ and the road surface $\left(\mathbf{P}_{\mathrm{r}}\right.$ ), respectively (Fig. 4a). Notably, point $\mathbf{P}_{\mathrm{f}}$ have z-coordinates greater than $\mathbf{P}_{\mathrm{r}}$ ones. Subsequently, Delaunay triangulation was applied across the mixed $\mathbf{P}_{\mathrm{f}}$ and $\mathbf{P}_{\mathrm{r}}$ data set (Fig. 4b). Road edge points $\left(\mathbf{P}_{\mathrm{cr}}\right)$ are those within $\mathbf{P}_{\mathrm{f}}$ that are vertices of triangles connecting both $\mathbf{P}_{\mathrm{f}}$ and $\mathbf{P}_{\mathrm{r}}$ (points Fig. 4c).

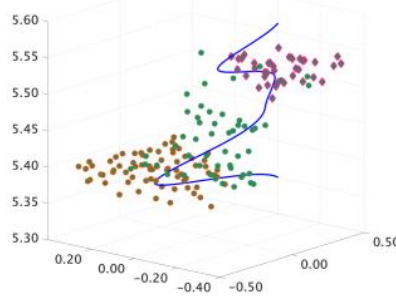

a) Extract $\mathbf{P}_{\text {f }}$ and $\mathbf{P}_{\text {r }}$ from the larger data set

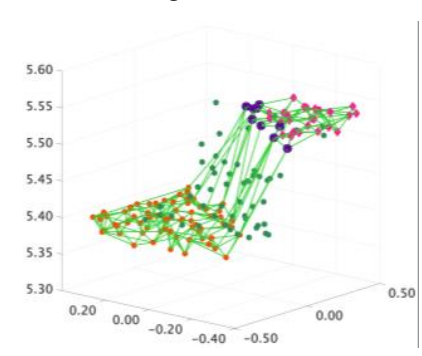

c) Extract $\mathbf{P}_{\mathrm{cr}}$

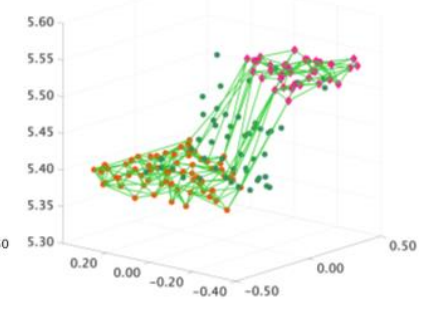

b) Generate Delaunay triangles from $\mathbf{P}_{\mathrm{f}}$ and $\mathbf{P}_{\mathrm{r}}$

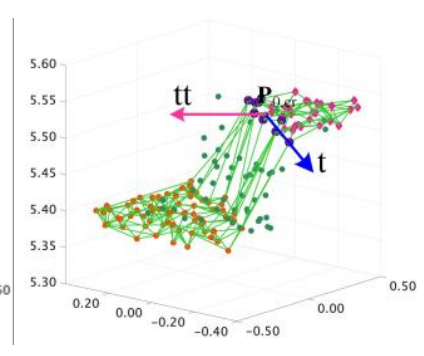

d) Features of each $\mathbf{C}_{\mathrm{rc}}$
Figure 4. Extract points of a road edge and compute features of each $\mathbf{C}_{\mathrm{rc}}$

In reality, there are unreal-curb cells, for example the cells represent planter boxes along the footpath, along the road having 
features similar to the curb cell. Thus, Step 3 groups curb points belonging to the road edge and remove incorrect road edge points. The process starts by employing a least square fitting to fit a line in $3 \mathrm{D}$ space though the $\mathbf{P}_{\mathrm{cr}}$ of each $\mathrm{C}_{\mathrm{rc}}$. From this, each curb cell has the road direction $(\mathrm{t})$ as a line direction vector, the road side direction (tt) which is an orthogonal vector of $\mathrm{t}$, indicating the road surface, and the centroid $\left(\mathbf{P}_{0, \mathrm{cr}}\right)$ of the $\mathbf{P}_{\mathrm{cr}}$ (Fig. 4d). Then, a cell-based region segmentation is used to group the $\mathbf{C}_{\mathrm{rc}}$. The segmentation started with an arbitrary $\mathrm{C}_{\mathrm{rci}}$ to search its neighbour curb cells $\mathrm{C}_{\mathrm{rcj}}$ by using a range search with the radius (r) by $3 \mathrm{~m}$. This selected radius was used to ensure (1) the curb cells on the same road edge can be checked, if there is a gap between $\mathbf{C}_{\mathrm{rc}}$ due to occlusion or disabled access ramps, and (2) the curb cell on the opposite side of the road may not include. The $\mathrm{C}_{\mathrm{rcj}}$ was considered as part of the same group with $\mathrm{C}_{\mathrm{rci}}$, if (1) the deviation angle of the two directional vectors $t_{i}$ of $C_{\text {rci }}$ and $t_{j}$ of $\mathrm{C}_{\mathrm{rcj}}$ was less than the angle threshold by 10 degrees, and (2) the road side direction vectors were in the same direction, which implies the sign between $\mathrm{tt}_{\mathrm{i}}$ of $\mathrm{C}_{\mathrm{rci}}$ and $\mathrm{tt}_{\mathrm{j}}$ of $\mathrm{C}_{\mathrm{rcj}}$ is positive. Finally, small segments in terms of length can be eliminated as they may not represent road curbs. To achieve this, a length threshold by $3.0 \mathrm{~m}$ was applied.

\section{EXPERIMENTAL TEST, RESULTS AND DISCUSSIONS}

To evaluate the proposed method, a portion of a $1.5 \mathrm{~km}^{2}$ ALS data of the city centre of Dublin, Ireland acquired in 2015 was used, which has a point density in horizontal about 335 points $/ \mathrm{m}^{2}$ (Laefer et al., 2017). The high density point cloud comes from a special flight planning designed to acquire maximum the vertical data of the building facades (Truong-Hong et al., 2015), which including a low flight attitude, large overlap between flight strips, and 2 flight paths (north-east to south-west and north-west to south-east). A $200 \mathrm{~m} \times 100 \mathrm{~m}$ section of the ALS data was selected to demonstrate the proposed method. The total number of points included in that portion of the point cloud was about 7.9 million points.

In this test, a cell size of $1.0 \mathrm{~m}$ was used as the terminal condition for cell decomposition, which is similar found from work by Aljumaily et al. (2015). The height of the road curb was assumed to vary between $10 \mathrm{~cm}$ and $30 \mathrm{~cm}$. Additionally, in segmenting the curb cells, the threshold angle of 10 degrees was used, while the length threshold of the road edge segment was set at $3.0 \mathrm{~m}$, which is less than the minimum lane width.

Point extraction results of the road edges are shown in Figure 5. With the Figure 5a input data in, the cell-based filtering algorithm roughly extracted ground points containing points of the road edges (Figure 5b). The road edge points derived from the proposed method are illustrated in Figure $5 \mathrm{c}$ as black dots.

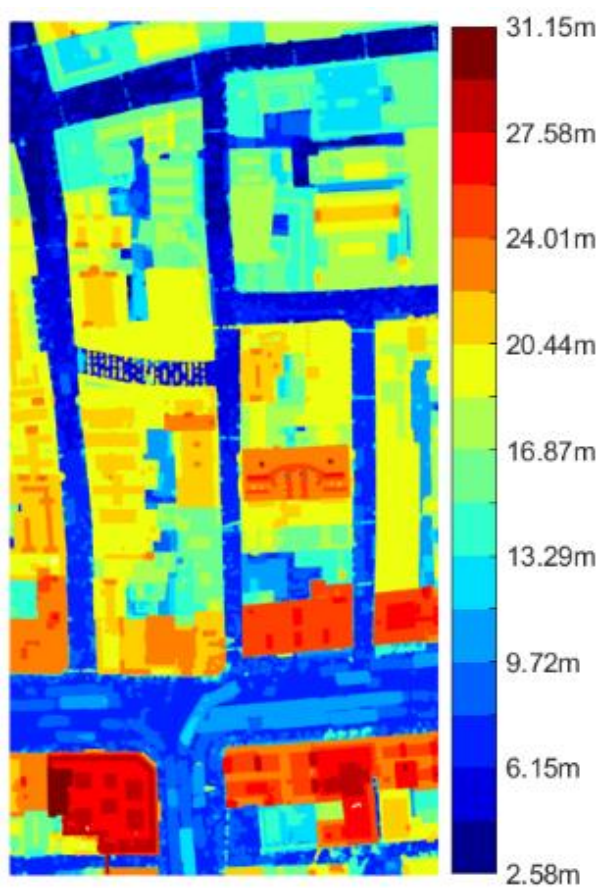

a) Input ALS data

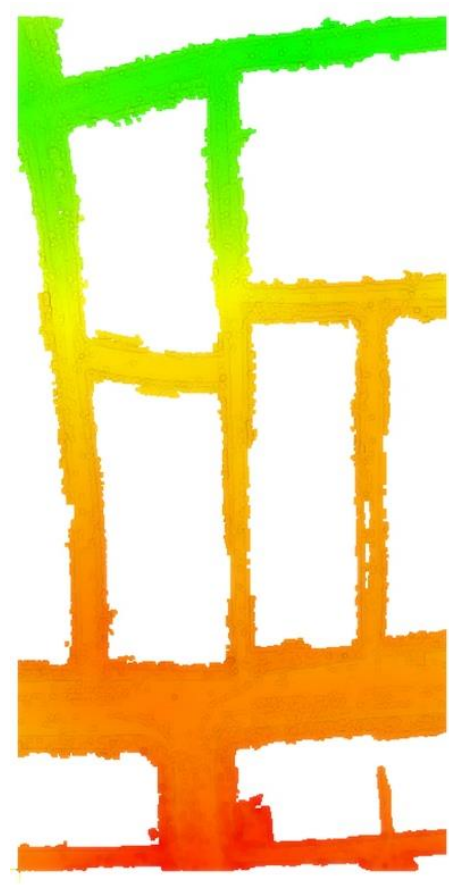

b) Ground points

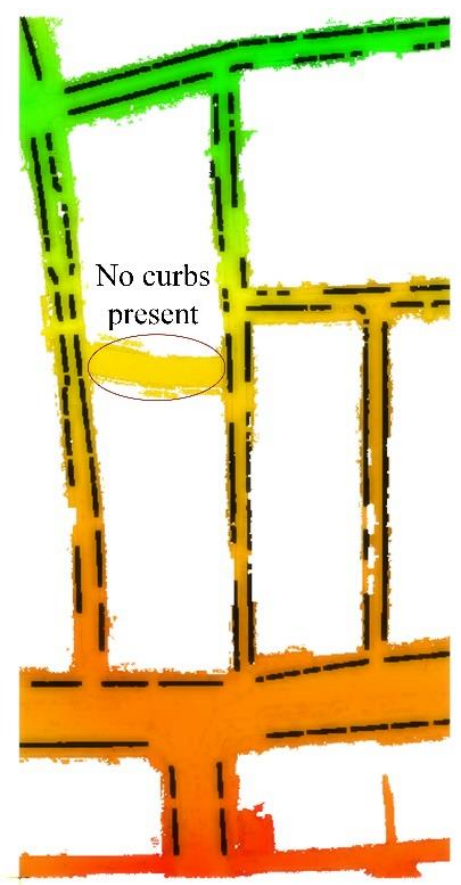

c) Road edge points overlaid on ground points

Figure 5. Results of road edge point extraction from selected ALS data in Dublin centre

Experimental tests showed that the proposed cell-based filtering method is simple but can give sufficient ground points containing the point clouds of both footpath and road surfaces (Figure 5b). Additionally, a visualisation evaluation showed the proposed method can extract proper data points of the road edges. Missing the road edge points occurred at locations where is no real road curbs available, which can be disabled accessibility through a slope. In this case, by using the KDE, the proposed method failed to detect real road curbs at these locations because there is often one local peak in the KDE. Moreover, the points of the road edges at the corners were eliminated because the curb cell segments do not satisfy the condition of the road edge segment length. That is at the corner, the road edge often has a small radius, which implies the deviation between directional vectors of adjacent curb cells exceeding the angle threshold. Thus, the segmentation of the curb cells were return short segments at these locations and to be eliminated by the length threshold. This problem will be sort out in future work. Importantly, missing points of the road edges are only a small portion of the road length, which may not affect to further processing based on the road edge points (e.g. road edge reconstruction or accessibility identification). 
To evaluate the accuracy of the extracted points belonging to the road edges, a 2D footprint of Dublin city was used as the ground truth. By adopting a comprehensive evaluation strategy both to determine discrepancy of road extraction from ALS data developed by Truong-Hong et al. (2015), which was evaluated accuracy of both location and the position of extracted roads, two evaluated metrics to measure accuracy of the extracted points of the road edges. The first entitled the distance error is defined as the orthogonal distance from extracted points of the road edges to the road edge in the ground truth. However, as only $2 \mathrm{D}$ ground truth is available, the distance error is here computed from $\mathrm{x}$ - and $y$ - coordinate of the points only. The second is an overlap ratio measured the length of the road edges can generated directly from their extracted points, which is the length of the extracted road edge points divided by ones of the road edges in the ground truth. For this task, the road edge points were projected onto the ground truth, and then the length of the road edge was calculated by assuming the road edge as a straight line.

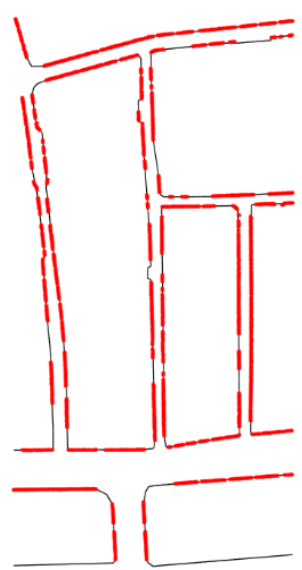

a) Extracted road edge points overlaid on the ground truth

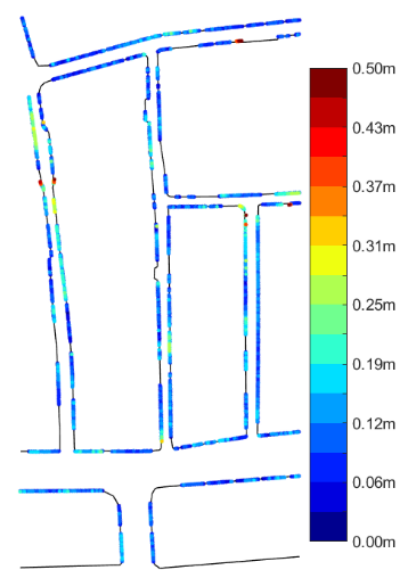

b) Distance errors of extracted points of the road edges
Figure 6. Distance errors of extracted points of the road edges

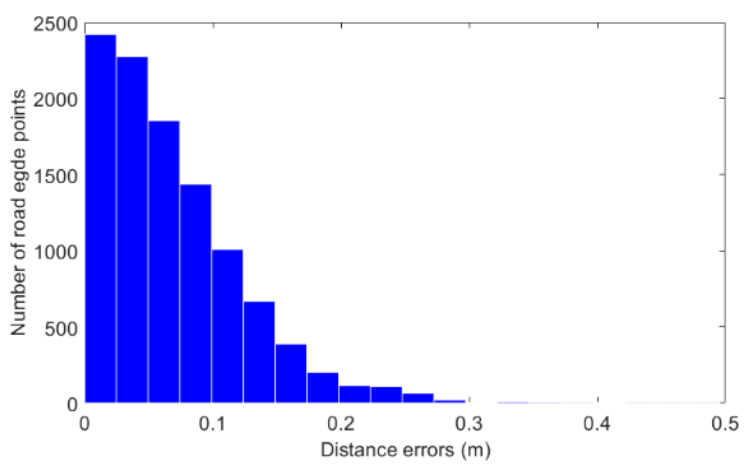

Figure 7. Distribution of the distance errors

The distance error for each extracted point along the road edge is shown in Figure 6b. There is a visible skew distribution of the distance error (Figure 7). Although the maximum distance error is $0.495 \mathrm{~m}$, the average distance errors are only $0.07 \mathrm{~m}$. In fact, about $59 \%$ of the road edge points have a distance error of less than $0.07 \mathrm{~m}$. Moreover, the evaluation also showed that the proposed method can give an overlap ratio up to $73.2 \%$. With this overlap ratio, the road edge is likely to be reconstructable from these points.

Finally, the proposed method was implemented in MATLAB scripts, and processed the selected study data set on Dell Precision Workstation with a main system configuration as follows: Intel(R) Xeon(R) W-2123 CPU @ 3.6GHz with 32GB RAM. The processing time is 200.6 seconds including executing time for ground point extraction by 163.8 seconds.

\section{CONCLUSIONS}

This paper presents a new hierarchical method for extracting road curbs from ALS data. The quadtree was employed to subdivide a bounding box enclosing the point cloud into smaller cells, in which a cell size of $1 \mathrm{~m}$ was used as the terminal condition. Cell height analysis and a surface-based filtering technique were combined to roughly extract the ground points. Following this, an analysis of the number of local maximum peaks of KDE was conducted, which were based on the z-coordinates of the data points within each cell. Footpath and road surface points within the cells as identified as curb cells were extracted, as being points located within the two outermost peaks of the KDE. Subsequently, Delaunay triangulation was applied from which the road edge points were identifiable as vertices of triangles connected both to footpath and road surface points. Next, cellbased segmentation was used to group the curb cells based on deviations of a direction and road side direction vectors of the curb cells. Finally, road edge segments having lengths smaller than a pre-specified length threshold (e.g. $3 \mathrm{~m}$ ) were reclassified as non-road edge. The proposed method tested on ALS data points of a selected $200 \mathrm{~m} \times 100 \mathrm{~m}$ city centre area of Dublin, Ireland. The points of the road edges in the study area were successfully extracted. The average distance error and overlap ratio of the extracted road edge points were respectively $0.07 \mathrm{~m}$ and $73.2 \%$ when comparing to the road edges from the ground truth. Next, a 3D model of the road edges could be reconstructed and a comprehensive evaluation strategy implemented to identify accuracy of location and the position of the extracted road edges.

\section{ACKNOWLEDGEMENTS}

This work was funded by the generous support of the European Commission through H2020 MSCA-IF, "BridgeScan: Laser Scanning for Automatic Bridge Assessment", Grant 799149.

\section{REFERENCES}

Aljumaily, H., Laefer, D.F. and Cuadra, D., 2015. Big-data approach for three-dimensional building extraction from aerial laser scanning. Journal of Computing in Civil Engineering, 30(3), p.04015049.

Clode, S., Kootsookos, P.J. and Rottensteiner, F., 2004. The automatic extraction of roads from LIDAR data. ISPRS Twentieth Annual Congress (Vol. 35, pp. 231-236).

Ferchichi, S., \& Shengrui, W. (2005, 11-14 Sept. 2005). Optimization of cluster coverage for road centre-line extraction in high resolution satellite images. IEEE International Conference on Image Processing 2005, Vol. 2, pp. II-201.

Guan, H., Li, J., Yu, Y., Wang, C., Chapman, M., \& Yang, B. (2014). Using Mobile Laser Scanning Data for Automated Extraction of Road Markings. ISPRS Journal of Photogrammetry and Remote Sensing, 87, 93-107. doi: j.isprsjprs.2013.11.005

Hinks, T., Carr, H. and Laefer, D.F., 2009. Flight optimization algorithms for aerial LiDAR capture for urban infrastructure 
model generation. Journal of Computing in Civil Engineering, 23(6), pp.330-339.

Ibrahim, S., \& Lichti, D. (2012). Curb-based Street Floor Extraction from Mobile Terrestrial LiDAR Point Cloud. ISPRSInternational Archives of the Photogrammetry, Remote Sensing and Spatial Information Sciences, XXXIX-B5, 193-198.

Jaakkola, A., Hyyppä, J., Hyyppä, H., \& Kukko, A., 2008. Retrieval Algorithms for Road Surface Modelling Using LaserBased Mobile Mapping. Sensors, 8(9), 5238.

Kumar, P., McElhinney, C. P., Lewis, P., \& McCarthy, T., 2013. An Automated Algorithm for Extracting Road Edges From Terrestrial Mobile LiDAR Data. ISPRS Journal of Photogrammetry and Remote Sensing, 85, 44-55. doi: 10.1016/j.isprsjprs.2013.08.003.

Lacoste, C., Descombes, X., \& Zerubia, J., 2005. Point processes for unsupervised line network extraction in remote sensing. IEEE Transactions on Pattern Analysis and Machine Intelligence, 27(10), 1568-1579. doi:10.1109/TPAMI.2005.206.

Laefer, D., Abuwarda, S., Vo, A., Truong-Hong, L., Gharibi, H., 2017. 2015 Aerial Laser and Photogrammetry Survey of Dublin City Collection Record. URL https://geo.nyu.edu/catalog/nyu_2451_38684. Last accessed 30 July 2017.

Laefer, D.F. and Truong-Hong, L., 2017. Toward automatic generation of 3D steel structures for building information modelling. Automation in Construction, 74, pp.66-77.

Lafarge, F., \& Descombes, X., 2010. Geometric Feature Extraction by a Multimarked Point Process. IEEE Transactions on Pattern Analysis and Machine Intelligence, 32(9), 15971609. doi:10.1109/TPAMI.2009.152

Miraliakbari, A., Hahn, M., \& Sok, S. (2015). Automatic Extraction of Road Surface and Curbstone Edges from Mobile Laser Scanning Data. The International Archives of Photogrammetry, Remote Sensing and Spatial Information Sciences, 40(4), 119.

Pu, S., Rutzinger, M., Vosselman, G., \& Oude Elberink, S., 2011. Recognizing basic structures from mobile laser scanning data for road inventory studies. ISPRS Journal of Photogrammetry and Remote Sensing, 66(6, Supplement), S28S39. doi: j.isprsjprs.2011.08.006

Qiu, K., Sun, K., Ding, K., \& Shu, Z. (2016). A Fast and Robust Algorithm for Road Edges Extraction From LiDAR Data. ISPRS-International Archives of the Photogrammetry, Remote Sensing and Spatial Information Sciences, 693-698.

Serna, A., \& Marcotegui, B. (2013). Urban accessibility diagnosis from mobile laser scanning data. ISPRS Journal of Photogrammetry and Remote Sensing, 84, 23-32. doi: 10.1016/j.isprsjprs.2013.07.001.

Seibert, A., Hähnel, M., Tewes, A. and Rojas, R., 2013. Camera based detection and classification of soft shoulders, curbs and guardrails. In Intelligent Vehicles Symposium (IV), 2013 IEEE (pp. 853-858).
Vo, A.V., Truong-Hong, L. and Laefer, D.F., 2015, July. Aerial laser scanning and imagery data fusion for road detection in city scale. IGARSS, 2015 IEEE International (pp. 4177-4180).

Smadja, L., Ninot, J., \& Gavrilovic, T. (2010). Road extraction and environment interpretation from LiDAR sensors. IAPRS, $38,281-286$.

Truong-Hong, L., \& Laefer, D. F., 2015. Quantitative evaluation strategies for urban 3D model generation from remote sensing data. Computers \& Graphics, 49, 82-91. doi: 10.1016/j.cag.2015.03.001

Truong-Hong, L., Laefer, D., Bisheng, Y., Ronggang, H. and Jianping, L., 2015. IQPC 2015 Track: Evaluation of Automatically Generated 2D Footprints from Urban LiDAR Data. International Archives of the Photogrammetry, Remote Sensing \& Spatial Information Sciences, XL-3/W3, 2015, pp.527-534

Vosselman, G., \& Zhou, L., 2009. Detection of curbstones in airborne laser scanning data. The International Archives of the Photogrammetry, Remote Sensing and Spatial Information Sciences, 38, 111-116.

Wang, W., Yang, N., Zhang, Y., Wang, F., Cao, T., Eklund, P., 2016. A review of road extraction from remote sensing images. Journal of Traffic and Transportation Engineering, 3(2), 271282, doi: j.jtte.2016.05.005.

Zhu, P., Lu, Z., Chen, X., Honda, K., \& Eiumnoh, A., 2004. Extraction of City Roads Through Shadow Path Reconstruction Using Laser Data. Photogrammetric Engineering \& Remote Sensing, 12, 1433-1440.

Zhou, L., \& Vosselman, G., 2012. Mapping Curbstones in Airborne and Mobile Laser Scanning Data. International Journal of Applied Earth Observation and Geoinformation, 18, 293-304. doi: j.jag.2012.01.024

Yang, B., Fang, L., \& Li, J. (2013). Semi-automated Extraction and Delineation of 3D Roads of Street Scene from Mobile Laser Scanning Point Clouds. ISPRS Journal of Photogrammetry and Remote Sensing, 79, 80-93. doi:10.1016/j.isprsjprs.2013.01.016 